\title{
A System for Simulation of Tip Evolution Under Field Evaporation
}

\author{
B. P. Geiser, D. J. Larson, S. S. A. Gerstl, D. Reinhard, T. F. Kelly, T. J. Prosa, and J. D. Olson \\ Imago Scientific Instruments Corp., 5500 Nobel Dr., Madison, WI 53711, USA
}

An improved method to numerically simulate the endform evolution of atom probe specimens has been developed in order to support reconstruction algorithm research. Similar to work described in [1], the system consists of a three-dimensional Poisson simulation and includes methods for choosing which atoms of the specimen to evaporate and a trajectory integrator to produce data that can be postevaporation processed, for example, by the IVAS system [2]. The current simulation uses a finite difference algorithm which specifies that atoms assigned to the tip are treated as part of the applied boundary conditions. The iteration used to solve for the potential is a standard multi-grid technique [3]. The simulation supports a wide range of tip geometries with included planar, spherical and cylindrical sub-structures. All components can be simulated with independently controllable compositions, evaporation fields and lattice structures.

By utilizing electrostatic simulation grids finer than the lattice spacing we now are able to simulate simple cubic, body-centered cubic and face-centered cubic (FCC) structures. Finer grid spacing enables more accurate calculation of electrostatic potentials (and thus electric fields) above the specimen surface, as shown in Fig. 1. We use a mesh refinement of $2 \mathrm{X}$ in each spatial direction for the simulations shown here. In simulated field desorption images, Fig. 2a, we observe realistic pole and zone-line structures typical of FCC systems and reproduce zone line doubling somewhat proportional to that observed in high quality Al desorption data [5-6], Fig. 2b. These features were earlier described by developing heuristic arguments based on the geometry of nearest neighbors [4]. By examining the local electrostatic environments and trajectories of the atoms involved, we are able to directly investigate the causes of various aberrations classically observed in atom probe data.

With these improvements in the electrostatic simulation of specimen evolution and in the resulting trajectories we are approaching the ability to meaningfully compare detailed differences between calculation and experiment in desorption map aberrations. This model then constitutes a feedforward model for image simulation similar to that in common practice in phase contrast imaging in transmission electron microscopy. We anticipate that this will be a powerful tool in understanding the relative importance of various physical effects occurring during evaporation.

\section{References}

[1] F. A. Vurpillot, F., et al., Journal of Microscopy 196 (3) (1999) 332-336.

[2] T. Kunicki, et al., Vac. Nan. Conf. 50 ${ }^{\text {th }}$ IFES, Tech. Digest, $19^{\text {th }}$ Intl, (2006) 535.

[3] W. Hackbusch, Multi-Grid Methods and Applications, $2^{\text {nd }}$ ed., Springer-Verlag, 2003.

[4] A. J. W. Moore, Phil. Mag. A 43 (3) (1981) 803.

[5] A. R. Waugh, et al., Surf. Sci. 61 (1976) 109.

[6] Data courtesy of Leigh Stephenson and Michael Moody, The University of Sydney. 


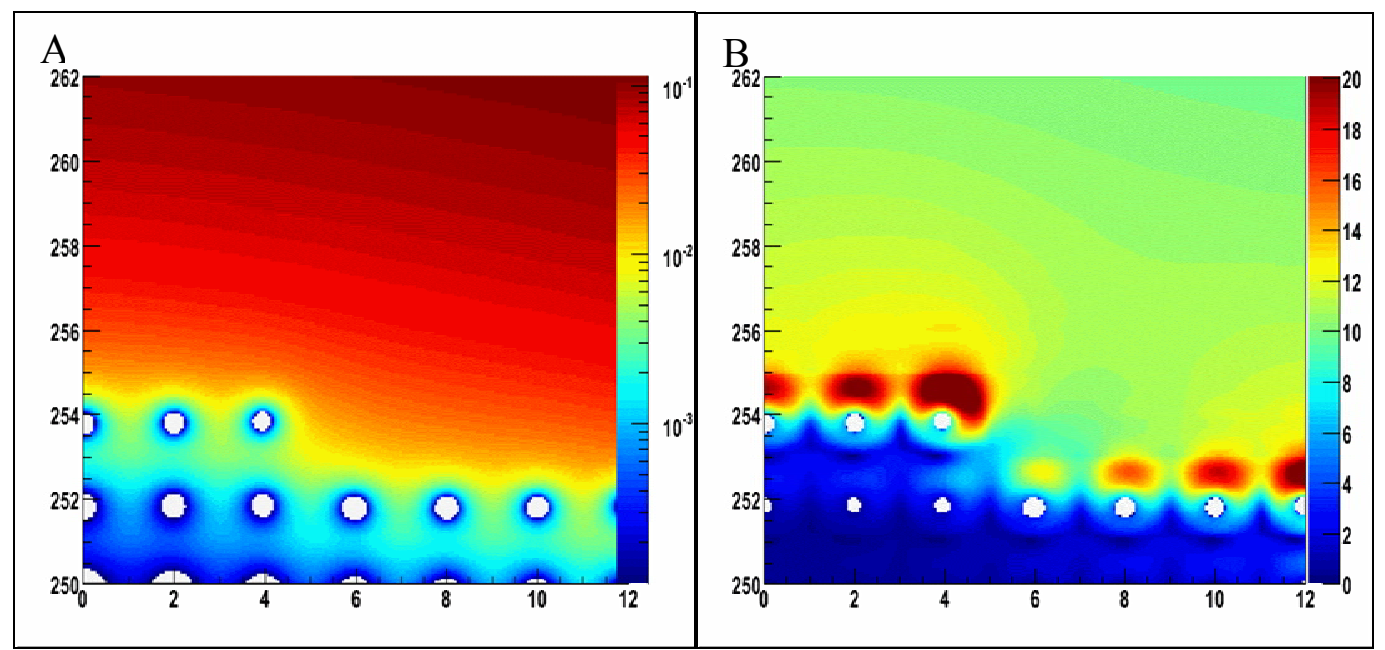

Fig. 1. A) The calculated electric potential and B) electric field strength close to a terrace edge.
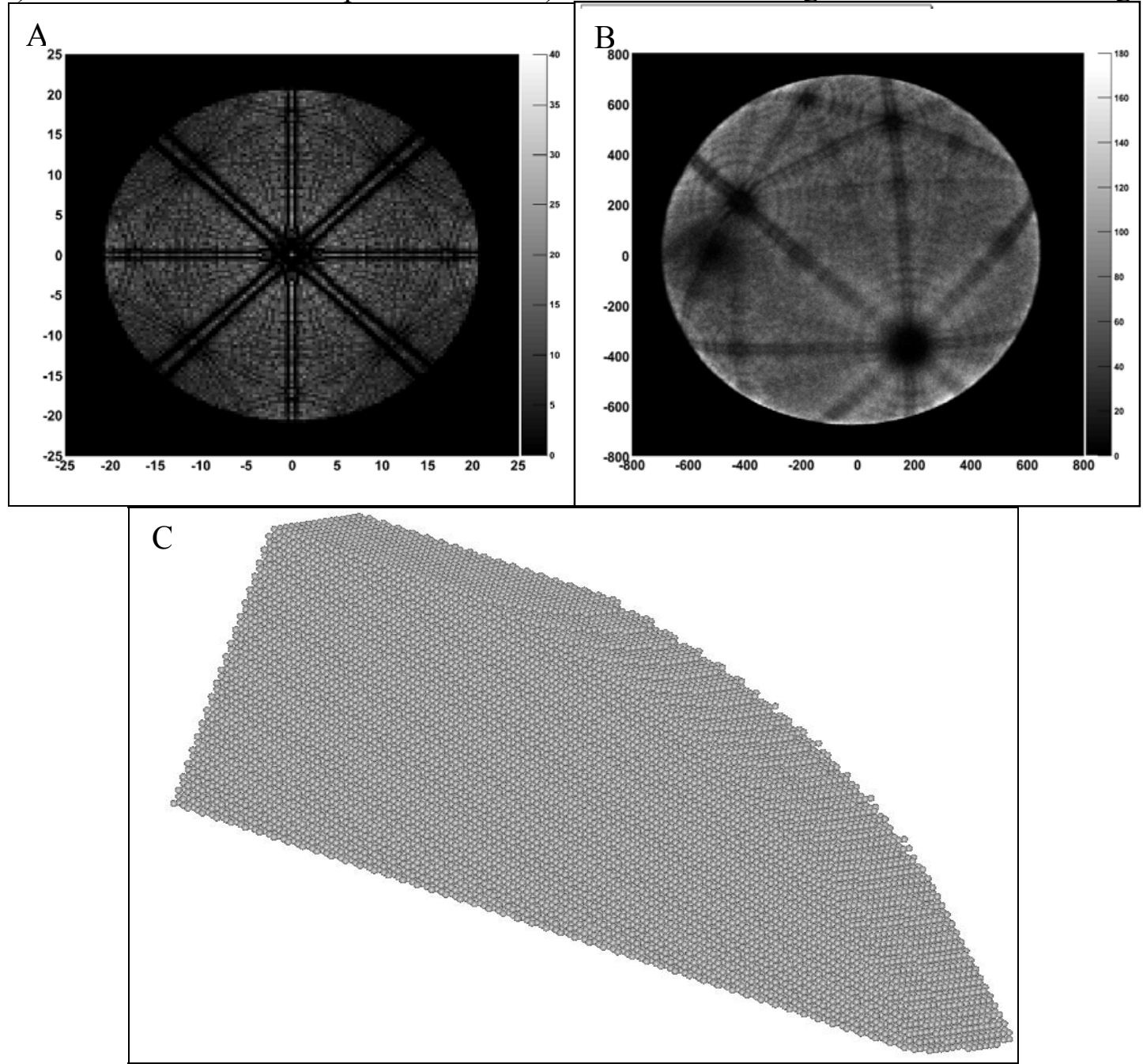

Fig. 2. A) Simulated hitmap resulting from evaporation of a pure system with fcc crystal-structure. B) Hitmap resulting from evaporation of $\mathrm{Al}$ at $15 \mathrm{~K}$. [6] C) A small segment of the evaporated simulation endform along the 010 zone-line. 